\title{
Case Study: Pulsed Electromagnetic Field (PEMF) Therapy Relieves Pleural Effusion Following Open Heart Surgery
}

\author{
Magda Havas* \\ Trent School of the Environment, Trent University, Canada
}

JSubmission: May 09, 2017; Published: May 30, 2017

*Corresponding author: Magda Havas, BSc., Ph.D., Trent School of the Environment, Trent University, Peterbrough, ON, K9J 7B8, Canada, Email: mhavas@trentu.ca

\begin{abstract}
Sixty-one year old female experienced difficulty breathing that became progressively worse following triple bypass open-heart surgery. She was readmitted to hospital and received oxygen therapy. X-rays indicated a large pleural effusion of the left lung that was increasing in size. She then received two 20-minute treatments with pulsed electromagnetic field (PEMF) therapy spaced one day apart. She noticed a significant improvement in breathing following the second treatment but not the first. A follow-up X-ray indicated a "dramatic" reduction in the pleural effusion that the radiologist assumed was due to therapeutic thoracocentesis. No thoracocentesis was performed. This case study indicates that PEMF therapy can be used to reduce a large pleural effusion after just two non-invasive treatments leading to an immediate improvement in symptoms verified by X-rays.
\end{abstract}

\section{Opinion Piece}

Pulsed electromagnetic field (PEMF) therapy has been used for decades to promote healing of non-union bone fractures and damaged soft tissue [1-3]. It has been shown to be effective at increasing circulation, reducing inflammation, and alleviating pain $[4,5]$. Currently transcranial PEMF therapy is being tested as an alternative to anti-depressants for those for whom drug therapy is ineffective [6].

Many different PEMF devices are available and a growing number of them have been approved by Health Canada and allowed into the United States as wellness devices. To date, there are no peer-reviewed studies documenting the effectiveness of PEMF on pleural effusions.

\section{Case Study}

A 61-year old female had triple bypass open heart surgery on July 18, 2013 and went home 9 days later. At home she had difficulty breathing and on July 31st was re-admitted to hospital where she received oxygen therapy. X-rays revealed that she had a large pleural effusion of the left lung. On August 3rd she returned home but still had breathing difficulties. X-rays taken on August 2, 9, and 20 indicated an increase in size of the pleural effusion (Plate 1). On September 16 and 17, she had two 20-minute treatments with ONDAMED, a PEMF therapeutic device that is based on pulse biofeedback. She noticed a significant improvement in breathing following the second but not the first treatment. X-rays taken on October 18 showed a "remarkable" improvement that the radiologist assumed was due to a therapeutic thoracocentesis. No thoracocentesis was performed. The only explanation is that the two PEMF treatments initiated the improvement that was subjectively observed and confirmed by X-rays.

Below are direct quotes from the radiologist.

August 9, 2013: Large left sided pleural effusion with compressive ateleclasis in the left lower lobe. No significant change when compared with the patient's previous examination on August 2, 2013 (Plate 1).

August 20, 2013: Comparison is made with the previous of 9 August 2013. There is a mild increase in the size of the large left pleural effusion and this occupies approximately the inferior two thirds of the left hemi thorax. Fluid is also seen tracking in the superior aspect of the left major fissure. The left upper lung including the right lung is clear and the right costophrenic angle is well defined (Plate 2).

October 18, 2013: The study is done on a patient who is seen to have a large left sided pleural effusion on an examination of August 20, 2013 (Plate 3). 

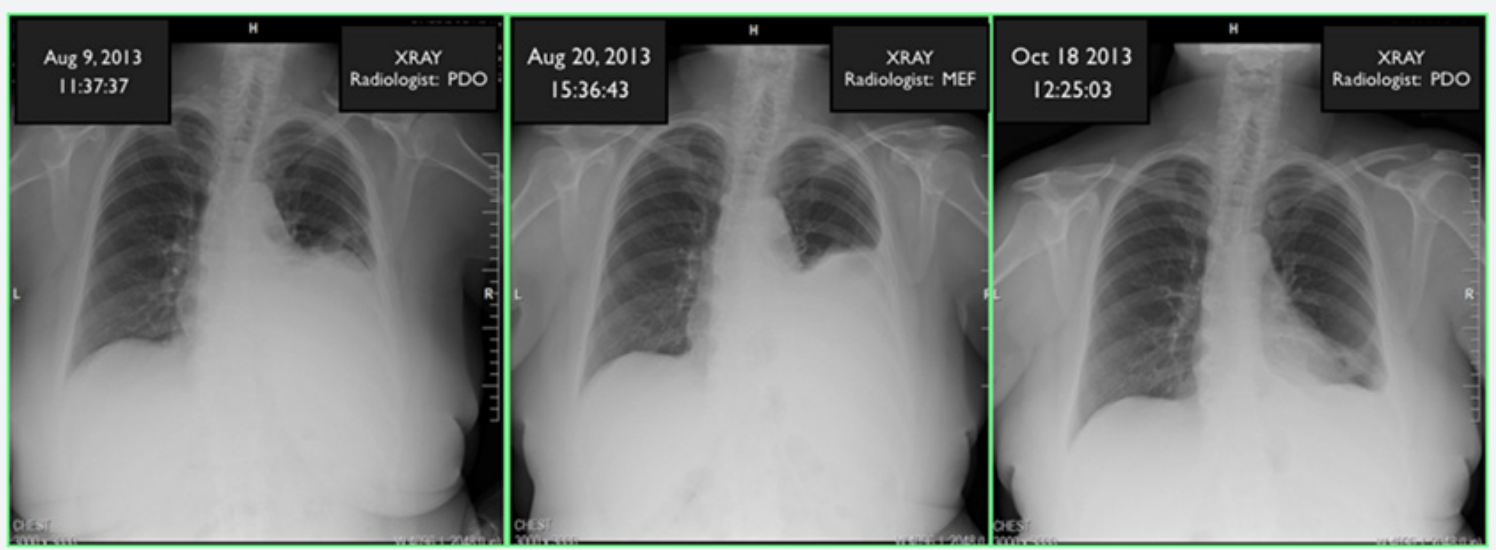

Plate 1: Sixty-one year old female experienced a large left sided pleural effusion in lower left lobe following open heart surgery. No change in size from x-ray taken on August 2, 2013.

Plate 2: Since August 9, 2013, there has been a mild increase in the left pleural effusion. Fluid is seen tracking the superior aspect of the left major fissure.

Plate 3: Left-sided pleural effusion shows a dramatic reduction in size when compared to August 20th, 2013.

The patient has had a prior CABG. The heart is within normal limits in size. The pulmonary vasculature is normal. The left sided pleural effusion shows a dramatic reduction in size when compared to the examine of August 20th. This is presumably due to a therapeutic thoracocentesis. [NOTE: no therapeutic thoracocentesis was performed] The left sided pleural effusion is now a small left sided pleural effusion. Mild compressive atelectasis is seen in the base of the left lung.

\section{Conclusion}

Two 20-minutes sessions of PEMF therapy one day apart were effective at reducing a pleural effusion in a 61-year old female following open heart surgery. Her ability to breath improved following the second treatment and the reduction in the pleural effusion is obvious in her chest x-rays.

\section{References}

1. Bassette CAL and RO Becker (1962) Generation of Electric Potentials by Bone in Response to Mechanical Stress, Science 137(3535): 10631064.
2. Pilla AA (2006) Mechanisms and therapeutic applications of timevarying and static magnetic fields. In: Barnes F, Greenebaum B (Eds.), Handbook of Biological Effects of Electromagnetic Fields. ( $3^{\text {rd }}$ edn), CRC Press, Florida, United States, p. 18.

3. Markov MS (2007) Pulsed electromagnetic field therapy history, state of the art and future. The Environmentalist 27(4): 465-475.

4. Wade B (2013) A review of pulsed electromagnetic field (PEMF) mechanisms at a cellular level: a rationale for clinical use. American Journal of Health Research 1(3): 51-55.

5. Strauch B, C Herman, R Dabb, LJ Ignarro, AA Pilla (2009) EvidenceBased Use of Pulsed Electromagnetic Field Therapy in Clinical Plastic Surgery. Aesthetic Surgery Journal 29(2): 135-143.

6. Martiny KM Lunde, P Bech (2010) Transcranial Low Voltage Pulsed Electromagnetic Fields in Patients with Treatment-Resistant Depression. Biol Psychiatry 68(2): 163-169.
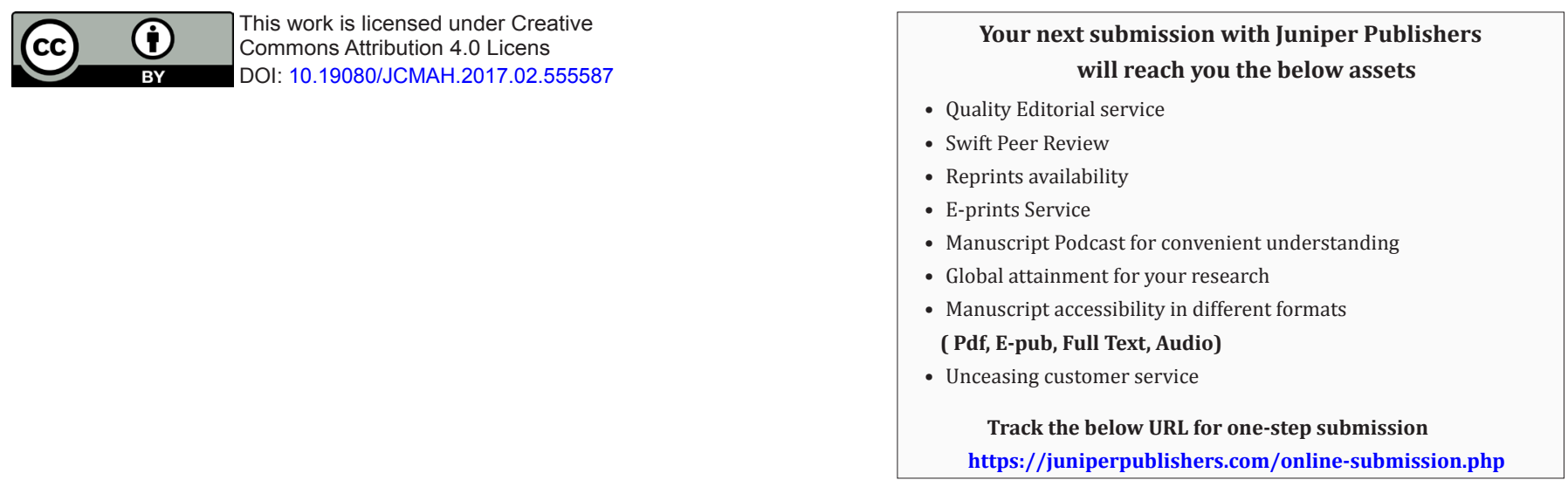